\title{
Remote password authentication with smart cards
}

\author{
C.-C. Chang \\ T.-C. Wu
}

Indexing terms: Codes and decoding, Cryprography, Security

\begin{abstract}
A remote password authentication scheme based on the Chinese remainder theorem is proposed. The scheme can verify the remote password without verification tables. In the initial phase, the password generation centre generates and assigns a password corresponding to each user. The ideas of smart cards and the identitybased signature scheme introduced by Shamir are employed in this phase. Each user possesses a smart card for later login and authentication. In the login phase, the user submits the identity and password associated with the smart card. In the authentication phase, the system verifies the remotely submitted password to check if the login request is accepted or rejected. A signature scheme and communication timestamps are provided in the authentication phase against the potential attacks of replaying a previously intercepted login request.
\end{abstract}

\section{Introduction}

The importance of ensuring privacy and security is acute because of the rapid progress and prevalence of multiuser computing environments. Various types of security mechanisms have been employed to preclude information in the computer systems being disclosed, destroyed, altered or copied by unauthorised users. The password authentication schemes are the best-known and the most accepted of these mechanisms by contemporary computer systems $[4,5,13]$.

In the conventional password authentication schemes, each user has an identity (ID) and a secret password (PW). When a user requests entry to the system, the correct ID and PW should be submitted so as to successfully pass the system authentication. One straightforward approach to achieve the verification is to directly store and maintain a directory of user IDs and PWs in the system. Such an approach cannot eliminate the threat of revealing passwords in the directory.

To overcome the weakness of storing IDs and PWs directly in the system, some approaches $[1,5,7-12,15]$ have been proposed which encode the passwords as test patterns or verification tables instead of the plain passwords directory. The whole system may be insecure and

Paper 7947E (C2), first received 13th September 1990 and revised form 15 th January 1991

C.-C. Chang is with the Institute of Computer Science and Information Engineering, National Chung Cheng University, Chiayi, Taiwan 62107 , Republic of China

T.-C. Wu is with the Institute of Computer Science and Information Engineering, National Chiao Tung University, Hsinchu, Taiwan 30043 Republic of China

IEE PROCEEDINGS-E, Vol. 138, No. 3, MAY 199I broken down if the test patterns or verification tables are modifed by malicious users in these approaches. Chang and $\mathrm{Wu}[2]$ proposed a password authentication scheme without verification tables. In their scheme, the user is burdened with requests for additional information other than the ID and PW in the login stage.

Consider only the remote password authentication schemes in remote access systems. The privacy and security problems are threatened by potential attacks from the remote terminals, along the communication links, as well as the system itself [13]. Lamport [11] proposed a scheme which protects against attacks of replaying previously intercepted requests. This scheme is insecure if the encrypted password stored in the centre is modified by an intruder. Denning [5] proposed another method by using the signature scheme of a public-key cryptosystem. That system also maintains verification tables. A remote password authentication system has the following characteristics:

(i) The system does not need to store or maintain verification tables.

(ii) The login request should be verified easily and quickly.

(iii) The scheme is secure against attacks of replaying previously intercepted request.

Inspired by Shamir's identity-based signature scheme [18], a remote password authentication scheme based on the Chinese remainder theorem is proposed. A brief review of Shamir's identity-based signature scheme is stated before presenting the scheme.

\section{Review of Shamir's identity-based signature} scheme

The identity-based cryptosystems and the signature scheme proposed by Shamir [18] enable any pair of users to communicate securely and to verify each other's signature without exchanging private or public keys and without keeping key directories. It also eliminates use by a third party. Shamir's identity-based signature scheme is first described.

Let $n, p, q, e$ and $d$ be parameters in the RSA scheme [16], where $p$ and $q$ are large primes and $n=p q$, $e d=1 \bmod (p-1)(q-1)$. The system publishes $e$ and $n$. $p, q$ and $d$ are kept secret. That is, $d$ is known only to the key generation centre. The $i$ th user's secret key $K_{i}$ is computed by the centre as

$$
K_{i}=\left(I D_{i}\right)^{d} \bmod n
$$

where $I D_{i}$ is the $i$ th user's identity. The $i$ th user may sign a message $m$ and the signature of $m$ can be verified by anyone who knows $I D_{i}$ as

$$
\begin{aligned}
& t=r^{e} \bmod n \\
& s=K_{i} r^{S(t, m)} \bmod n
\end{aligned}
$$


where $r$ is a random number selected by the user and $f$ is a one-way function. The pair $(t, s)$ is the signature of $m$. To anyone who knows $I D_{i}$, the signature of $m$ can be easily verified using the test

$$
s^{e}=I D_{i} t^{f(t, m)}(\bmod n)
$$

For physical implementation, the secret key generated by the centre is issued to the user in the form of smart card when the user first registers to the system. A smart card is an IC processor which can efficiently perform computational operations [14]. The smart card possessed by the user contains a microprocessor, and I/O port, a RAM, a ROM with the user's secret key and programs for generating and verifying the signature [18]. Any pair of users can verify each other's signature easily and quickly.

The merits of Shamir's identity-based signature scheme is that it is simple and secure. It is also suitable for remote access systems. It is weak against the potential attack of replaying previously intercepted authentication keys if an intruder knows the identity $I D_{i}$ and eavesdrops on the signature message $s$ and $t$.

A new remote password authentication scheme is presented. The concept of timestamps [6] is employed to avoid attacks by using the strategy of replaying previously intercepted passwords.

\section{Proposed scheme}

Since the scheme uses the Chinese remainder theorem (CRT), the theorem is described. Given $2 n$ positive integers $m_{1}, m_{2}, \ldots, m_{n}$, and $r_{1}, r_{2}, \ldots, r_{n}$, a constant $C$ can be found such that

$$
C \equiv r_{1} \bmod m_{1}, C \equiv r_{2} \bmod m_{2} \ldots, C \equiv r_{n} \bmod m_{n}
$$

if $m_{i}$ and $m_{j}$ are relatively prime for all $i \neq j$ [17].

The CRT can be applied to encrypt the plaintext and decrypt the ciphertext. Let $d_{1}, d_{2}, \ldots, d_{m}$ be $m$ large relatively prime numbers and $a_{1}, a_{2}, \ldots, a_{\mathrm{m}}$ be $m$ plain messages. The encrypted data is

$$
C \equiv a_{i} \bmod d_{i} \quad i=1,2, \ldots, m
$$

The value $a_{i}$ can be recovered computing

$$
\begin{aligned}
& \quad a_{i}=C \bmod d_{i} \\
& \text { Let } D=d_{1} d_{2}, \ldots, d_{m} \text { and } b_{i} \text { satisfy }
\end{aligned}
$$

$$
\left(\frac{D}{d_{i}}\right) b_{i} \equiv 1 \bmod d_{i} \quad i=1,2, \ldots, m
$$

The encryption keys can be computed as

$$
e_{i}=\left(\frac{D}{d_{i}}\right) b_{i} \quad i=1,2, \ldots, m
$$

By the CRT

$$
C=\left(\sum_{i=1}^{m} e_{i} a_{i}\right) \bmod D
$$

The proposed remote password authentication scheme can be divided into three phases. In the initial phase, when a user registers to the system, the password generation centre generates a password (PW) for the user according to the presented identity (ID). The password is returned to the user through a very secure channel or by hand. A smart card, storing the information used by the login and authentication phases, is constructed and delivered to the user. In the login phase, the user attaches the smart card to a terminal and submits the ID and PW.
In the authentication phase, the system verifies the remotely submitted password to check if the login request is accepted or rejected.

Let $d_{1}, d_{2}, \ldots, d_{m}$ be large relatively prime numbers and $D=d_{1} d_{2}, \ldots, d_{m}$, where $d_{1}, d_{2}, \ldots, d_{m}$ are known only to the password generation centre. Let $g$ be a pseudorandom number generating function and $f$ be a one-way function. The algorithm of $g$ should be kept secret in the system, i.e., anyone who knows $x$ cannot predict the value of $g(x)$, and the value of $g(x)$ is less than $D . D$ and $f$ can be made public.

Initial phase: When a new user $U_{i}$ registers to the system, the identity $I D_{i}$ should be presented to the system. The password generation centre does the following:

(i) Generate a password $P W_{i}=\left(w_{i 1}, w_{i 2}, \ldots, w_{i m}\right)$, where

$$
w_{i j}=g\left(I D_{i}\right) \bmod d_{j} \quad j=1,2, \ldots, m
$$

and $m$ is predetermined integer, say $m=5$.

(ii) Deliver a smart card, which contains the information $\left\{f,\left(e_{1}, e_{2}, \ldots, e_{m}\right), d\right\}$, to the user $U_{i}$.

The smart cards possessed by all users are the same. The smart card contains a microprocessor which can perform arithmetic operations quickly, an I/O port, a RAM, a ROM in wihch is stored the algorithmic description of the one-way function $f$ and parameters $\left(e_{1}, e_{2}, \ldots, e_{m}\right)$ and $D$, and programs for generating signature and authenticating message.

Login phase: For login the system, $U_{i}$ first attaches the smart card to a terminal. The $I D_{i}$ and $P W_{i}$ are then keyed in. The smart card performs the following tasks:

(i) Generate a random vector $\left(r_{1}, r_{2} \ldots, r_{m}\right)$.

(ii) Let $P W_{i}=\left(w_{i 1}, w_{i 2}, \ldots, w_{i m}\right)$. Compute

$$
\begin{aligned}
t & =\left(\sum_{i=1}^{m} e_{i} r_{i}\right) \bmod D \\
s & =\left(w_{i 1}, w_{i 2}, \ldots, w_{i m}\right)+\left(r_{1}, r_{2}, \ldots, r_{m}\right) f(t, T) \\
& =\left(x_{i 1}, x_{i 2}, \ldots, x_{i m}\right)
\end{aligned}
$$

where $T$ is the login current date and time used as timestamp.

(iii) Construct the authenticating message $C=\left\{I D_{i}, t\right.$, $s, T\}$ and transmit $C$ to the system by communication link.

The pair $(t, s)$ computed by the smart card is used as the signature. The timestamp $T$ is employed to withstand potential attacks of replaying previously intercepted passwords. When the login procedure is finished, the authentication phase follows.

Authentication phase: Let $T^{\prime}$ be the date and time when the system receives the message $C$ sent by the login user $U_{i}$. After receiving the message $C$, the system verifies the remote login with the following steps:

(i) Check if the format of $I D_{i}$ is correct. If it is incorrect then reject the login request.

(ii) Test if $T^{\prime}-T \leqslant \Delta T$, where $\Delta T$ is the legal time interval for transmission delay. If it is false, then reject the login request.

(iii) Encrypt $s$ by keys $\left(e_{1}, e_{2}, \ldots, e_{m}\right)$. Then test if the result is equal to $\left(g\left(I D_{i}\right)+t f(t, T)\right) \bmod D$. If it is true, then accept the login request; otherwise, reject the login request. 
Examining step (iii) of the authentication phase carefully, it is found that the result of encrypting $s$ is equal to

$$
\begin{aligned}
& \left(\sum_{j=1}^{m} e_{j} x_{i j}\right) \bmod D \\
& \quad=\left(\sum_{j=1}^{m} e_{j}\left(w_{i j}+r_{j} f(t, T)\right)\right) \bmod D \\
& =\left(\left(\sum_{j=1}^{m} e_{j} w_{i j}\right) \bmod D+\left(\sum_{j=1}^{m} e_{j} r_{j}\right) f(t, T)\right) \bmod D \\
& =\left(g\left(I D_{i}\right)+t f(t, T)\right) \bmod D
\end{aligned}
$$

\section{Security analysis and discussions}

The secrecy of the CRT is based on the factoring of the large number $D$. As pointed out in Reference 3 , if one knows $\left(C, w_{j}\right)$ and $\left(C^{\prime}, w_{j}^{\prime}\right)$ pairs computed from

$$
\begin{gathered}
w_{j}=C \bmod d_{j} \\
w_{j}^{\prime}=C^{\prime} \bmod d_{j} \\
C-w_{j}=Q d_{j} \\
C^{\prime}-w_{j}^{\prime}=Q^{\prime} d_{j}
\end{gathered}
$$

then

for some $Q$ and $Q^{\prime}$.

It has a high probability of revealing $d_{j}$ by finding the greatest common divisor of $C-w_{j}$ and $C^{\prime}-w_{j}^{\prime}$. In the initial phase of generating passwords, the password $P W_{i}$ has a one-to-one correspondence to the returned value of a secure pseudorandom number generating function $g$ with identity $I D_{i}$ as the seed of $g$. It can therefore prevent any two conspirational users with known $\left(I D_{i}, P W_{i}\right)$ and $\left(I D_{j}, P W_{j}\right)$ pairs from maliciously revealing $d_{j}$. It should be noted that the users with different identities may have the same passwords if the function $g$ is not a one-to-one mapping.

An intruder may try to masquerade as $U_{i}$ by replaying previously intercepted message $C=\left(I D_{i}, t, s, T\right)$. To pass step (iii) in the authenticating phase, the intruder must change the timestamp $T$ to $T^{*}$ such that $T^{\prime}-T^{*} \leqslant \Delta T$. Once the timestamp $T$ is changed, either $t$ or $s$ has to be changed. So the scheme can withstand potential attacks with the strategy of replaying a previously intercepted login request

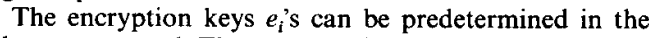
scheme presented. The computational complexities of the scheme is examined in each phase. Let $T_{g}$ be the time required for the pseudorandom number generating function $g$, and let $T_{f}$ be the time required for the one-way function $f$. The time complexities in each stage are listed below.

Initial phase:

Time for password generation

$$
=T_{g}+(m \text { modular operations })
$$

Login phase:

Time for computing $s=(m$ multiplications $)$

$$
\begin{aligned}
& +\{(m-1) \text { additions }\} \\
& +(1 \text { modular operation })
\end{aligned}
$$

Time for computing $t=T_{f}+$ ( $m$ multiplications)

$$
+ \text { ( } m \text { additions) }
$$

\section{Authentication phase:}

Time for encrypting $s=(m$ multiplications $)$

$$
\begin{aligned}
& +\{(m-1) \text { additions }\} \\
& +(1 \text { modular operation })
\end{aligned}
$$

Time for verification $=T_{g}+T_{f}+(1$ multiplication $)$

$$
\begin{aligned}
& +(1 \text { addition }) \\
& +(1 \text { modular operation }) \\
& +(1 \text { comparison })
\end{aligned}
$$

The login and authentication phases can be performed easily and quickly by applying the smart card. However the user of the system cannot choose his password freely. If a user's password has to be changed, for some security considerations, a new identity has to be reassigned to the user and the old identity should not be used by new users in the initial phase.

\section{Conclusions}

A remote password authentication scheme which does not use a directory of passwords or verification tables is presented. The scheme is very useful in remote access systems or computer networks with remote login under insecure communication links. By employing the concept of timestamps, the scheme can withstand attacks which use the strategy of replaying previously intercepted login request.

A disadvantage of the scheme is that a very secure channel is required for the return of the password to the registering user. The users cannot freely choose their passwords. The problem of allowing users to freely choose their passwords in the authentication system without storing verification tables still remains open.

\section{Acknowledgments}

The authors would like to thank the referees for useful comments which have improved the presentation of this paper.

\section{References}

1 CHANG, C.C., and WU, L.H.: 'A password authentication scheme based upon Rabin's public-key cryptosystems', Proc. Int. Conf. Systems Management ' 90 , Hong Kong, June 1990, pp. 425-429

2 CHANG, C.C., and WU, T.C.: 'A password authentication scheme without verification tables', Proc. 8th lASTED Int. Symp. Applied Informatics, February 1990, Innsbruck, Austria, pp. 202-204

3 DAVIDA, G.I., WELLS, D.L., and KAM, J.B.: 'A database encryption system with subkeys', $A C M$ Trans. Database Syst., 1981, 6, (2) pp. $312-328$

4 DAVIES, D.W., and PRICE, W.L.: 'Security for computer networks' (John Wiley, New York, 1984)

5 DENNING, D.E.: 'Cryptography and data security' (AddisonWesley, Massachusets, 1982)

6 DENNING, D.E., and SACCO, G.M.: 'Timestamps in key distribu tion protocols', Commun. ACM, 1981, 24, (8), pp. 533-536

7 EVANS, A. Jr., KANTROWITZ, W., and WEISS, E.: 'A user authentication scheme not requiring secrecy in the computer', Commun. ACM $1974,17,(8)$, pp. 437-442

8 FEISTEL, H., NOTZ, W.A., and SMITH, J.L.: 'Some cryptographic techniques for machine to machine data communications', Proc IEEE, 1975, 63, (11), pp. 1545-1554

9 HWANG, T.Y.: 'Passwords authentication using public-key encryption', Proc. Int. Carnahan Conf. Security Technology, Zurich, Switzerland, October 1983, pp. 35-38

10 LAIH, C.S., HARN, L., and HUANG, D.: 'Password authentication using quadratic residues', Proc. 1988 Int. Computer Symp., Taipei, Taiwan, December 1988, pp. 1484-1489 
11 LAMPORT, L.: 'Password authentication with insecure communication', Commun. ACM, 1981, 24, (11), pp. 770-772

12 LENNON, R.E., MATYAS, S.M., and MEYER, C.H.: 'Cryptographic authentication of time-invariant quantities', IEEE Trans. 1981, COM-29, (6), pp. 773-777

13 MORRIS, R., and THOMPSON, K.: 'Password security: a case study', Commun. ACM, 1979, 22, (11), pp. 594-597

14 OKAMOTO, E.: 'Identity-based information security managemen system for personal computer networks', IEEE J. Sel. Areas Commun., 1989, SAC-7, (2), pp. 290-294
15 PURDY, G.P.: 'A high security log-in procedure', Commun. $A C M$, $1974,17,(8)$, pp. $442-445$

16 RIVEST, R.L., SHAMIR, A., and ADLEMAN, L.: 'A method for obtaining digital signatures and public-key cryptosystems', Commun. $A C M, 1978,21$, (2), pp. $120-126$

17 SCHROEDER, M.R.: 'Number theory in science and communication' (Springer-Verlag, Berlin, 1983)

18 SHAMIR, A.: 'Identity-based cryptosystems and signature schemes' Proc. CRYPTO '84, Springer-Verlag, pp. 47-53 